\title{
Face Recognition Using Posterior Distance Model Based Radial Basis Function Neural Networks
}

\author{
S. Thakur ${ }^{1}$, J.K. Sing ${ }^{2, *}$, D.K. Basu ${ }^{2}$, and M. Nasipuri ${ }^{2}$ \\ ${ }^{1}$ Department of Information Technology, Netaji Subhas Engineering College, Kolkata, India \\ ${ }^{2}$ Department of Computer Science \& Engineering, Jadavpur University, Kolkata, India \\ jksing@ieee.org
}

\begin{abstract}
The success rate of a face recognition system heavily depends on two issues, mainly, i) feature extraction method and ii) choosing/designing of a classifier to classify a new face image based on the extracted features. In this paper, we have addressed both the above issues by proposing a new feature extraction technique and a posterior distance model based radial basis function neural networks (RBFNN). First, the dimension of the face images is reduced by a new direct kernel principal component analysis (DKPCA) method. Then, the resulting face vectors are further reduced by the Fisher's discriminant analysis (FDA) technique to acquire lower dimensional discriminant features. During classification, when the RBFNN is not so confident to classify a test image, we have introduced a statistical method called the posterior distance model (PDM) to resolve the conflict. The PDM is an approach, which takes a decision by integrating the outputs of the RBFNN and a distance measure. We call the new classifier the posterior distance model based radial basis function neural networks (PDM-RBFNN). The proposed method has been evaluated on the AT\&T database. The simulation results in terms of recognition rates are found to better than some of the existing related approaches.
\end{abstract}

Keywords: Face recognition, Radial basis function neural networks, Direct kernel principal component analysis, Fisher's discriminant analysis.

\section{Introduction}

Since the last decade, human face recognition is an active research area in the field of pattern recognition and computer vision due to its wide range of applications, such as identity authentication, access control, surveillance systems, security, etc. As a result, a number of methods have been proposed in the past and surveys in this area can be found in [1]-[4]. To achieve higher performance in face recognition, two issues are needed to be addressed: i) feature extraction process from a face image and ii) choosing or designing of a classifier to classify a new face image based on the extracted features. Till now, most of the efforts have been made to address the first issue by proposing various feature extraction methods [3]-[7]. Very few efforts have been

\footnotetext{
${ }^{*}$ Corresponding author.
} 
made to address both the above issues [8]-[10]. The present study addresses both the above issues to improve the performance of a face recognition system.

In this paper, we have addressed the feature extraction process and designing of a classifier to achieve higher success rate. A new kernel PCA, referred to as the direct kernel PCA (DKPCA) [11], is used for dimension reduction of the image data. The DKPCA explicitly maps an input image nonlinearly into a feature space spanned by the number of training samples and then computes the principal components directly in the mapped space. This method considers the structural information of the input images in the feature space for computation of principal components, leading to have higher discriminating power. It has been found that the DKPCA has higher discriminating power than the KPCA for face recognition [11]. After reducing the dimension of image data, the reduced feature vectors are further reduced using the Fisher's discriminant analysis (FDA) technique to acquire lower-dimension discriminant features. Since the DKPCA algorithm reduces the dimension of the image data considerably, the so-called 'small-sample-size' (SSS) problem does not arises while performing the FDA in the mapped space. We call the above feature extraction process using the DKPCA and FDA algorithms as the Direct Kernel Fisher Discriminant Analysis (DKFDA).

We have also designed a new classifier based on RBF neural networks (RBFNN) and a posterior distance model for face recognition. When the RBFNN is not so confident to recognize a test image, we have introduced a new statistical method called the posterior distance model (PDM) to resolve the conflict. The PDM is an approach, which takes a decision by integrating the top three outputs of the RBFNN and the distance measures between the test image and centres of the classes associated with the top three outputs of the RBFNN. The PDM improves the success rate for face recognition by reducing the numbers of false positives and false negatives. We call the new classifier the posterior distance model based radial basis function neural networks (PDM-RBFNN).

\section{Feature Extraction}

The face image features are extracted using a new direct kernel Fisher's discriminant analysis (DKFDA) algorithm. The DKFDA algorithm is implemented in two stages. In the first stage, high dimensional image data are reduced using direct kernel PCA method (DKPCA) [11]. The DKPCA method explicitly maps an input image nonlinearly into a feature space spanned by the rank of the training samples and then computes the principal components directly in the mapped space. Since the DKPCA considers the nonlinear structures of the input samples in the mapped feature space, the computed principal components have higher discriminating power. In the second stage, reduced image data are further reduced using Fisher's discriminant analysis (FDA) method. The FDA searches for those vectors in the underlying space that best discriminate among classes (rather than those that best describe the data). It finds an optimal subspace for classification that maximizes the ratio of the between-class scatter matrix and the within-class scatter matrix. We call the above technique, which uses the DKPCA and FDA, the direct kernel Fisher's discriminant analysis (DKFDA) for feature extraction. 


\section{Posterior Distance Model (PDM)}

The schematic diagram of the PDM-RBFNN is shown in Fig. 1. Let the output vector of the RBFNN for a test pattern $\mathbf{Z}_{\mathrm{i}}=\left(\mathrm{z}_{\mathrm{i} 1}, \mathrm{z}_{\mathrm{i} 2}, \ldots, \mathrm{z}_{\mathrm{im}}\right)$ is $\mathbf{C}_{\mathrm{i}}=\left(\mathrm{c}_{\mathrm{i} 1}, \mathrm{c}_{\mathrm{i} 2}, \ldots, \mathrm{c}_{\mathrm{iC}}\right)$, where $C$ is the total number of individuals in the database. Let top three outputs are $\mathrm{O}_{\mathrm{i} 1}, \mathrm{O}_{\mathrm{i} 2}$ and $\mathrm{O}_{\mathrm{i} 3}$, respectively. Let the classes associated with the top three outputs are $1_{1}, 1_{2}$ and $1_{3}$, respectively. We define a confidence factor $(C F)$ for classifying a test pattern as the standard deviation $(S D)$ of the top three outputs of the RBFNN. If the $C F$ is higher than a predefined threshold $\varepsilon$, the class of the test pattern is determined as the index of the output neuron, which produces maximum value. Otherwise, the PDM model determines its class by integrating the top three outputs of the RBFNN and distance measures as follows:

$$
\operatorname{class}\left(\boldsymbol{Z}_{\boldsymbol{i}}\right)=\arg \min _{\boldsymbol{l}_{1}, l_{2}, l_{3}}\left\{\boldsymbol{d}\left(\boldsymbol{Z}_{\boldsymbol{i}}, \boldsymbol{t}_{\boldsymbol{i}_{\boldsymbol{k}}}\right) *\left(1-\boldsymbol{o}_{\boldsymbol{i k}}\right)\right\}, \boldsymbol{k}=1,2,3
$$

where $\mathrm{d}\left(\mathbf{Z}_{\mathrm{i}}, \mathbf{t}_{\mathrm{ik}}\right)$ is a distance measure between the test pattern $\mathbf{Z}_{\mathrm{i}}$ and the mean training pattern $\mathbf{t}_{\mathrm{ik}}$ of the class associated with the top $k^{\text {th }}$ output of the RBFNN. In our experiments, we have used the Euclidean and Manhattan distance as the distance measure. The Manhattan distance is found to be better than the Euclidean distance. The above model, called the posterior distance model (PDM), is used to resolve the conflict arises when the RBFNN fails to recognize a test pattern confidently. The PDM improves the success rate by reducing the numbers of false positives and false negatives.

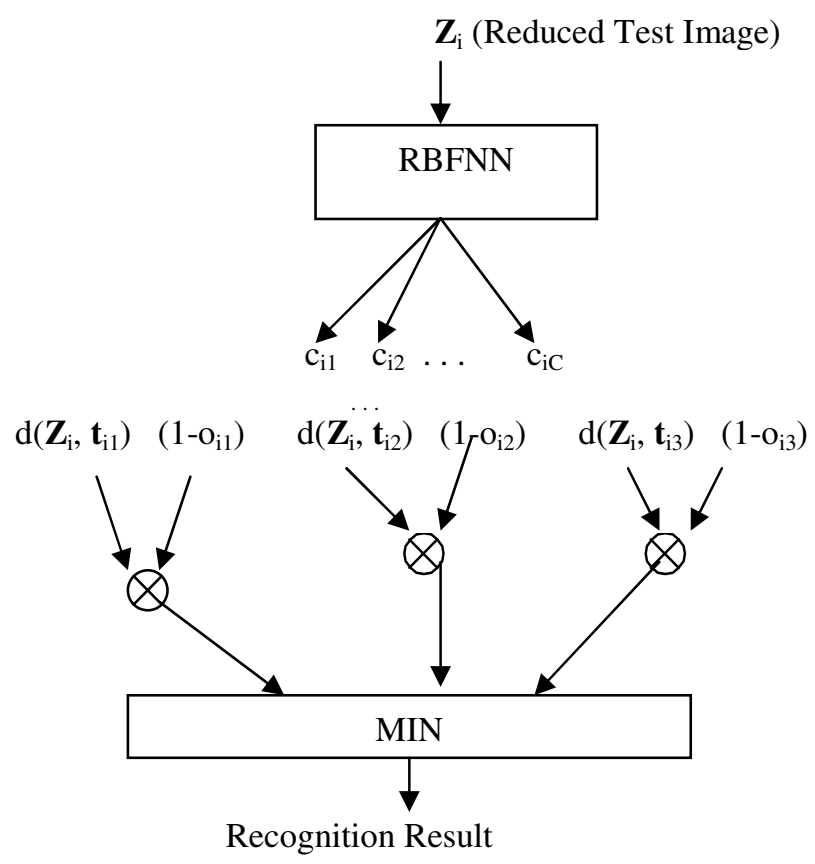

Fig. 1. Schematic diagram of the PDM-RBFNN 


\section{Experimental Results}

The performance of the proposed method has been evaluated on the AT\&T Laboratories Cambridge database (formerly ORL database) [12]. Several experiments have been carried out using three different methodologies; namely, i) randomly partitioning the database, ii) $\mathrm{N}$-fold cross validation test and iii) leave-one-out strategy.

\subsection{Randomly Partitioning the Database}

In these experiments, we have selected randomly $\mathrm{s}$ images per person from the database to form the training set. Remaining images form the corresponding test set. It should be noted that there is no overlap between the training and test images. In this way 10 different training and test sets have been generated for each value of $s$.

We have conducted several experiments by varying DKFDA features. Table 1 shows the best performances of the proposed method for $s=4,5$, and 6 . The best average recognition rates of $92.29 \%, 95.05 \%$ and $95.38 \%$ are achieved for $s=4,5$ and 6, respectively using Manhattan distance as the distance measure.

Table 1. Average recognition rates of the proposed methods by randomly partitioning the database

\begin{tabular}{|l|c|c|c|}
\hline \# samples/person, $s$ & 4 & 5 & 6 \\
\hline \# training samples & 160 & 200 & 240 \\
\hline \# test samples & 240 & 200 & 160 \\
\hline \# hidden layer neurons & 120 & 120 & 160 \\
\hline \# DKFDA features & 50 & 55 & 55 \\
\hline Average Recog. $(\%)$ & $\mathbf{9 2 . 2 9}$ & $\mathbf{9 5 . 0 5}$ & $\mathbf{9 5 . 3 8}$ \\
\hline Standard Deviation & 1.84 & 1.59 & 1.07 \\
\hline
\end{tabular}

\subsection{N-Fold Cross Validation Test}

In this study, the AT\&T database is randomly divided into ten-folds, taking one image of a person into a fold. Therefore, each fold consists of 40 images, each one corresponding to a different person. For ten-fold cross validation test, in each experimental run, nine folds are used for training and remaining one fold is used for testing. Therefore, training and test sets consist of 360 and 40 images, respectively in a particular experimental run. Several experiments are conducted by varying DKFDA features from 10-50. The best average recognition rate using the Manhattan distance as the distance measure is achieved using 20, 30, 40 and 50 DKFDA features. Table 2 shows the performances of the proposed method and the RBFNN without PDM model. The average recognition rate is found to be $97.75 \%$. It may be noted that performance of the proposed method is better than the RBFNN without the PDM model. 
Table 2. Recognition rates using $\mathrm{N}$-fold cross validation test on the ORL database

\begin{tabular}{|l|c|c|}
\hline \multicolumn{1}{|c|}{ Method } & Proposed method & $\begin{array}{c}\text { RBFNN } \\
\text { without PDM }\end{array}$ \\
\hline \# training samples & 360 & 360 \\
\hline \# hidden layer neurons & 200 & 200 \\
\hline Average Recog. (\%) & $\mathbf{9 7 . 7 5}$ & $\mathbf{9 7 . 5 0}$ \\
\hline Standard Deviation & 2.19 & 2.36 \\
\hline
\end{tabular}

\subsection{Leave-One-Out Strategy}

In this study, the experiments are carried out using the "leave-one-out" strategy. To classify a test image of a subject, the image is removed from the database of $N$ images and placed into a test set. Remaining $N-1$ images are used in the corresponding training set. In this way, experiments are performed $N$ times, removing one image from the database at a time. For the AT\&T database, we have performed 400 experimental runs for the database of 400 images. Table 3 shows the average recognition rate of the proposed system using 20 DKDFA features and 200 hidden layer neurons. We have achieved $97.50 \%$ average recognition rate.

Table 3. Experimental results using leave-one-out strategy

\begin{tabular}{|c|c|c|}
\hline $\begin{array}{c}\text { \# of hidden layer } \\
\text { neurons }\end{array}$ & $\begin{array}{c}\text { \# of DKFDA } \\
\text { features }\end{array}$ & $\begin{array}{c}\text { Average recog. } \\
(\%)\end{array}$ \\
\hline 200 & 20 & 97.50 \\
\hline
\end{tabular}

\section{Conclusion}

Success rate of a face recognition system is heavily depends on the feature extraction process and the choosing or designing of a suitable classifier. In this paper, we have described a direct kernel Fisher's discriminant analysis (DKFDA) technique and proposed a posterior distance model-based radial basis function neural networks (PDMRBFNN), to address the above two issues, respectively. The new system has been evaluated on the AT\&T face database. Experiments are carried out in three different strategies; namely, i) randomly partitioning the database, ii) $\mathrm{N}$-fold cross validation test and iii) leave-one-out strategy. The experimental results have demonstrated improved performance for the new system. The best average recognition rates of $92.29 \%, 95.05 \%$ and $95.38 \%$ have been achieved for $s=4,5$ and 6, respectively using Manhattan distance as the distance measure. In $\mathrm{N}$-fold cross validation test we have achieved $97.75 \%$ average recognition rate. Whereas in leave-one-out strategy, we have achieved $97.50 \%$ average recognition rate.

Acknowledgments. This work was partially supported by the CMATER and the SRUVM projects of the Department of Computer Science \& Engineering, Jadavpur University, Kolkata, India. The author, S. Thakur would like to thank Netaji Subhas 
Engineering College, Kolkata for providing computing facilities and allowing time for conducting research works. The author, D. K. Basu would also like to thank the AICTE, New Delhi for providing him the Emeritus Fellowship (F.No.: 151/RID/EF(13)/2007-08, dated 28-02-2008).

\section{References}

1. Samal, A., Iyengar, P.: Automatic recognition and analysis of human faces and facial expressions: A survey. Pattern Recognition 25, 65-77 (1992)

2. Chellapa, R., Wilson, C., Sirohey, S.: Human and machine recognition of faces: A survey. J. IEEE 83, 705-741 (1995)

3. Zhao, W., Chellappa, R., Phillips, P.J., Rosenfeld, A.: Face Recognition: A Literature Survey. ACM Computing Surveys 35, 399-458 (2003)

4. Tolba, A.S., El-Baz, A.H., El-Harby, A.A.: Face Recognition: A Literature Review. International Journal of Signal Processing 2, 88-103 (2006)

5. Lu, J., Plataniotis, K.N., Venetsanopoulos, A.N.: Face recognition using kernel direct discriminant analysis algorithms. IEEE Trans. Neural Networks 14, 117-126 (2003)

6. Yang, J., Frangi, A.F., Yang, J.-Y.: A new kernel Fisher discriminant algorithm with application to face recognition. Neurocomputing 56, 415-421 (2004)

7. Belhumeur, P.N., Hespanha, J.P., Kriegman, D.J.: Eigenfaces versus Fisherfaces: recognition using class specific linear projection. IEEE Trans. Pattern Anal. Mach. Intell. 23, $711-$ 720 (1997)

8. Er, M.J., Wu, S., Lu, J., Toh, H.L.: Face recognition with radial basis function (RBF) neural networks. IEEE Trans. Neural Networks 13, 697-710 (2002)

9. Sing, J.K., Basu, D.K., Nasipuri, M., Kundu, M.: Face recognition using point symmetry distance-based RBF network. Applied Soft Computing 7, 58-70 (2007)

10. Sing, J.K., Basu, D.K., Nasipuri, M., Kundu, M.: High-speed face recognition using selfadaptive radial basis function neural networks. To appear in Neural Computing \& Application, Springer, Heidelberg

11. Sing, J.K., Basu, D.K., Nasipuri, M., Kundu, M.: Direct kernel PCA with RBF neural networks for face recognition. In: IEEE TENCON 2008, Hyderabad, India (2008)

12. ORL face database. AT\&T Laboratories, Cambridge, UK, http: / /www.uk.research.att.com/facedatabase.html 